\title{
CARACTERIZAÇÃO DA ARBORIZAÇÃO URBANA EM TRÊS AMBIENTES NA CIDADE DE MONTES CLAROS, MG
}

\author{
Maria das Dores Magalhães Veloso', Lílian de Lima Braga1', Priscyla Maria Silva Rodrigues², \\ Marianna Rodrigues Santos ${ }^{2}$, Weslane Oliveira Miranda1, Diego Oliveira Brandão ${ }^{3}$ \\ Yule Roberta Ferreira Nunes ${ }^{1}$
}

\section{RESUMO}

A arborização urbana é um fator importante que proporciona um ambiente físico saudável aos centros urbanos. O presente trabalho teve como objetivo inventariar e comparar a diversidade de espécies vegetais utilizadas na arborização de três ambientes na área urbana da cidade de Montes Claros através de levantamento florístico, análise de parâmetros da arborização, da distribuição dos indivíduos e da diversidade através do índice de Shannon $(H)$. Foi encontrado um total de 1605 indivíduos, pertencentes a 24 famílias botânicas. O bairro Major Prates apresentou maior riqueza, com 46 espécies e maior abundância com 951 indivíduos, seguido do bairro Morada do Parque, com 38 espécies e 486 indivíduos, e das Praças, com 33 espécies e 168 indivíduos. As praças obtiveram maior diversidade $\left(H^{\prime}=2,9\right)$, maiores indivíduos e maiores copas. Todos os parâmetros analisados diferiram entre os ambientes estudados, exceto para a área livre do passeio e o espaçamento entre os indivíduos. O bairro Major Prates apresentou uma média de raiz exposta maior que o Morada do Parque e também uma menor média de distância que ultrapassa a fiação. Assim, os três ambientes possuem diferenças marcantes em seus padrões de arborização.

Palavras-chave: Áreas verdes; Centros urbanos; Diversidade de espécies; Planejamento urbano.

Recebido em 13.03.2013 e aceito em 27.02.2015

1 Laboratório de Ecologia Vegetal, Departamento de Biologia Geral, Universidade Estadual de Montes Claros, CP 126, CEP 30401-089, Montes Claros, MG. Emails: dora.velos013@gmail.com, lilianlbraga@yahoo.com.br, lanebio10@hotmail.com, yule.nunes@unimontes.br

2 Programa de Pós-Graduação em Botânica, Departamento de Biologia Vegetal, Universidade Federal de Viçosa, CEP 36570 000, Viçosa, MG. Emails: priscylamsr@gmail.com, mrodriguessantos@gmail.com.

3 INPA - Instituto Nacional de Pesquisas da Amazônia, Av. André Araújo, 2.936, Petrópolis, CEP 69067-375, Manaus, AM. Email: dbrandao13@gmail.com. 


\title{
CHARACTERIZATION OF URBAN AFFORESTATION IN THREE ENVIRONMENTS IN MONTES CLAROS CITY, MG
}

\begin{abstract}
The urban forestry is an important factor that provides a physical healthy environment to urban centers. This study aimed to identify and compare the diversity of plant species used in forestry in three environments of the urban area of Montes Claros City through floristic survey, analysis of different afforestation parameters, the distribution of individuals and diversity through the Shannon index $(H)$. The total of 1,605 individuals belonging to 24 botanical families were found. Major Prates neighborhood had the highest richness, with 46 species and the highest abundance with 951 individuals, followed by Morada do Parque neighborhood, with 38 species and 486 individuals, and Squares with 33 species and 168 individuals. The Squares had higher diversity index $\left(H^{\prime}=2.9\right)$, larger individuals and bigger tree canopy. All parameters differ among the environments, except for the free ride area and the spacing between individuals. The Major Prates neighborhood had a larger mean of exposed root that the Morada do Parque and also a smaller average distance that exceeds the wiring. Thus, the three environments have significant differences in their afforestation patterns.
\end{abstract}

Keywords: Green areas; Urban centers; Species diversity; Urban planning.

\section{INTRODUÇÃO}

A arborização urbana é constituída por toda a cobertura vegetal de porte arbóreo e arbustivo presente nas ruas, avenidas e praças das cidades (RODRIGUES et al., 2002). Segundo Gonçalves et al. (2004), a prática da arborização iniciou-se em Paris, no ano de 1660, com o intuito melhorar o aspecto cénico daquela cidade. Já no Brasil, esta prática teve início há pouco mais de 120 anos, nas ruas do Rio de Janeiro (DANTAS; SOUZA, 2004), passando a fazer parte do planejamento urbano de forma generalizada em várias regiões do país somente no final do século XIX (MENEGUETTI, 2003). 
A arborização requer uma atenção cada vez maior em função dos benefícios e até mesmo dos problemas que apresenta, em decorrência da presença das árvores no contexto da cidade. O planejamento urbano, ao estruturar a cidade, maneja os componentes da paisagem construída e entre estes o elemento vegetal (DANTAS; SOUZA, 2004), promovendo desta forma um equilíbrio entre as edificações e a vegetação.

A substituição dos elementos naturais por asfalto, edificações, vidros e estruturas metálicas, causou um impacto negativo na qualidade de vida das pessoas, por ter contribuído com o incremento de um clima quente e seco, além de ter provocado a impermeabilização do solo, o aumento da poluição sonora, visual e atmosférica (SILVA FILHO et al., 2005; GONÇALVES et al., 2004). Além disto, o crescimento desordenado das cidades, sem um planejamento de ocupação adequado, ocasiona problemas relacionados ao abastecimento de água, coleta de lixo e esgoto e pavimentação de ruas (SOLERA et al., 2005), o que também provoca alterações negativas ao ambiente, devido principalmente, à devastação da vegetação nativa. Sendo assim, o conhecimento e a análise das estruturas presente nas cidades e de suas funções, são necessários para o planejamento e administração das áreas urbanas, buscando melhoria na qualidade de vida dos seus habitantes (ROCHA et al., 2004).

Neste contexto, e pelos seus próprios objetivos, a arborização urbana assume importância singular, como um relevante fator na promoção de um ambiente mais ameno (ROCHA et al., 2004), além das funções ecológica e estética (RODRIGUES et al., 2002). Assim, a arborização proporciona sombreamento para pedestres e veículos, melhoria da qualidade do ar, redução da amplitude térmica, abrigo para a avifauna e o equilíbrio estético, amenizando a diferença entre o meio ambiente e outros componentes arquitetônicos como grandes avenidas, muros e prédios (SILVA FILHO et al., 2002). Entretanto, a arborização sem um planejamento prévio pode causar sérios problemas para a população, além de prejuízos econômicos, através rompimento de fios de alta-tensão, interrupções no fornecimento de energia elétrica, entupimento em redes e esgoto, obstáculos para circulação e acidentes envolvendo pedestres, veículos e ou edificações (SILVA FILHO et al., 2002; ZANIN, 2005). De acordo com Silva Filho et al. (2002), em muitas situações, o planejamento urbano deixa de incluir a arborização como uma ação a ser devidamente projetada. Isto permite, muitas vezes, que iniciativas particulares pontuais e desprovidas de conhecimento técnico atualizado, tomem espaço com plantios irregulares de espécies.

O conhecimento das espécies vegetais utilizadas na arborização de uma cidade torna-se indispensável para o planejamento e manejo desta arborização. Este procedimento pode fornecer ainda informações sobre a necessidade de poda, tratamentos fitossanitários, 
remoção e plantios de espécies adequadas (ROCHA et al., 2004). Neste sentido, o presente trabalho teve como objetivos inventariar e comparar a diversidade de espécies vegetais utilizadas na arborização de três ambientes urbanos na cidade de Montes Claros, no norte de Minas Gerais, bem como determinar as características das espécies utilizadas nesta arborização, como: área da copa, altura, espaçamento entre os indivíduos, exposição radicular, distância da rede elétrica e área livre do passeio. Desta forma, estes dados virão fornecer subsídios para a elaboração do planejamento urbano em que se utilizem espécies adequadas para a arborização de uma cidade.

\section{MATERIAIS E MÉTODOS}

\section{Área de estudo}

O município de Montes Claros está localizado no Norte do Estado de Minas Gerais, área de transição entre o Cerrado e a Caatinga, inserido na Bacia do Rio Verde Grande (IGAM, 2007). Possui uma população de 370.216 habitantes e sua área total perfaz $3.582,034 \mathrm{~km}^{2}$ (IBGE, 2012).

Para o presente estudo, foram selecionados três ambientes de estudos, sendo dois bairros da cidade de Montes Claros/MG: bairro Morada do Parque (coordenadas $16^{\circ} 45^{\prime}$

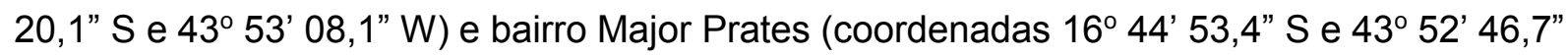
W), e o terceiro constituído das praças destes bairros.

O bairro Major Prates, urbanizado em 1964 e localizado na região Sul da cidade, possui, atualmente, uma população de 5.279 habitantes e conta com uma área de 1.775 .570 $\mathrm{km}^{2}$, fazendo limite com os bairros Augusta Mota, Morada do Parque, São Geraldo, Vargem Grande e Canelas (OLIVEIRA, 2003). Além disso, neste bairro, situam-se o Parque Municipal Milton Prates e o Zoológico Municipal, com extensa área verde e uma lagoa natural (OLIVEIRA, 2003), sendo os mesmos excluídos deste trabalho.

O bairro Morada do Parque é comparativamente mais novo que o Major Prates, urbanizado no ano de 1980. Apresenta uma área de $216.073 \mathrm{~km}^{2}$, com uma população de 2.219 habitantes. O bairro Morada do Parque faz limite com os bairros Ibituruna, Morada do Sol, Major Prates e Jardim São Geraldo (OLIVEIRA, 2003). 


\section{Levantamento florístico}

Para o levantamento da arborização, nos três ambientes: (1) bairro Major Prates, (2) bairro Morada do Parque e (3) praças, foram realizadas caminhadas semanais e sequenciais em todas as ruas e praças, perfazendo um censo, no período de fevereiro de 2005 a agosto de 2006. Nestas visitas, foram amostrados e identificados todos os indivíduos arbóreo-arbustivos, situados nas calçadas e no interior das praças, com CAP (circunferência a altura do peito, medida a $1,3 \mathrm{~m}$ do solo) $\geq 15 \mathrm{~cm}$. De todas as espécies encontradas foi coletado material botânico, posteriormente conduzido ao Laboratório de Ecologia Vegetal (LEVE) da Universidade Estadual de Montes Claros (UNIMONTES), e manipulados segundo as técnicas convencionais de herborização e depositados no Herbário Montes Claros (MCMG). Para aquelas espécies não identificadas in loco, a identificação foi feita através de consultas a especialistas, uso de literatura especializada e comparações com as exsicatas existentes no MCMG. O sistema de classificação das espécies adotado foi o Angiosperm Phylogeny Group II (APG II, 2003).

Para todas as plantas inventariadas foram ainda tomadas às seguintes medidas: (1) tamanho da copa - TC (cm), sendo este medido em duas direções, paralelo ao passeio e perpendicular ao mesmo; (2) tamanho das rachaduras no passeio - TRP (m), considerado como a medida da distância do tronco dos indivíduos até o ponto mais distante da rachadura ou da marca radicular no passeio, medida em duas direções: paralela e perpendicular ao passeio; (3) distância da fiação - DF ( $m$ ), medida através de uma estimativa visual da distância das copas das árvores aos fios de energia elétrica, linha telefônica e outros, considerada "positiva" quando a copa ultrapassava a fiação e "negativa" quando a copa encontrava-se abaixo da fiação; (4) altura da árvore - $H(m)$, estimada visualmente, tomando como referência altura dos postes de iluminação pública (aproximadamente $10 \mathrm{~m}$ ); (5) espaçamento ou distribuição dos indivíduos - EI (m): considerada através da medida de distância entre os indivíduos; e (6) área livre entre a árvore e o passeio - ALP (cm), através da medida da área sem pavimentação entre o indivíduo e o passeio, sendo realizada perpendicularmente a este. Estas medidas foram obtidas para estipular a distribuição, abundância e a área sombreada da arborização.

\section{Análise de dados}

Foi realizada uma análise descritiva dos indivíduos inventariados e obtenção do número total de indivíduos, espécies e famílias, além da quantificação e relação do número 
de indivíduos por rua, bairros e praças. A cobertura arbórea foi calculada através da fórmula da elipse $(A=\pi a b$; em que $a$ e $b$ são os raios, maior e menor, da elipse). As diferenças entre os parâmetros extraídos dos indivíduos (TC e $\mathrm{H}$ ), entre os locais amostrados foram verificadas através da Análise de Variância - ANOVA (ZAR, 1996). Para detectar a diferença do TRP, DF, El, ALP nos indivíduos dos dois bairros foi utilizado o Teste T (ZAR, 1996), já que estes parâmetros não foram obtidos nas praças. Para a análise da DF foram utilizados apenas os indivíduos que estavam do lado da rua em que se encontrava a fiação. A diversidade nos três ambientes amostrados foi calculada através do índice de diversidade de Shannon $\left(H^{\prime}\right)$ (KREBS, 1989).

\section{RESULTADOS E DISCUSSÃO}

Foram encontrados nos três ambientes estudados, 1606 indivíduos e 73 espécies pertencentes a 24 famílias botânicas (Tabela 1). Dentre as espécies encontradas, oito foram identificadas ao nível de gênero, uma em nível de família e seis espécies morfotipadas. 0 bairro Major Prates apresentou maior riqueza, com 46 espécies, e maior abundância, com 951 indivíduos, seguido do bairro Morada do Parque com 38 espécies e 486 indivíduos e das praças com 33 espécies e 168 indivíduos. Das espécies amostradas, 39 foram exclusivas de algum dos ambientes e 42 ocorreram com apenas um indivíduo.

As espécies mais abundantes, nos três ambientes estudados foram em ordem decrescente: Ficus benjamina (382 indivíduos), Licania tomentosa (363), Caesalpinia pluviosa (305) e Tabebuia impetiginosa (103). Além disto, espécies como Tabebuia impetiginosa, Terminalia catappa e Ficus benjamina foram mais representativas no bairro Major Prates, enquanto que Bauhinia variegata e Prosopis juliflora foram abundantes no bairro Morada do Parque e Jacaranda brasiliana e Delonix regia nas praças (Tabela 1). 
Tabela 1. Lista das famílias e espécies (abundância e riqueza), número de indivíduos por área e a caracterização da origem das espécies utilizadas na arborização de três ambientes da cidade de Montes Claros, MG. Origem Nativa $=1$, Exótica $=2$. Ambientes: $\mathrm{M} . \mathrm{P}=$ Major Prates, $\mathrm{M} . \mathrm{Pq}=$ Morada do Parque, $\mathrm{P}=$ Praças

Table 1. List of families and species (abundance and richness), number of individuals per area and the characterization of the species origin used in the urban afforestation in three areas in Montes Claros City, MG. Native $=1$, Exotic $=2$. Environments: $\mathrm{M} . \mathrm{P}=$ Major Prates, M.Pq = Morada do Parque, $\mathrm{P}=$ Praças

\begin{tabular}{|c|c|c|c|c|c|c|}
\hline \multirow{2}{*}{ Família } & \multirow{2}{*}{ Espécie } & \multirow{2}{*}{ Nome popular } & \multicolumn{4}{|c|}{0 Indiv./amb. } \\
\hline & & & & M. P & M. Pq & $\mathbf{P}$ \\
\hline \multirow{2}{*}{ Anacardiaceae } & Mangifera indica L. & Mangueira & 2 & 3 & 1 & - \\
\hline & Schinus mollis $\mathrm{L}$. & Aroeirinha-chorona & 2 & 2 & 10 & \\
\hline \multirow{3}{*}{ Apocynaceae } & \multirow{3}{*}{$\begin{array}{l}\text { Nerium oleander L. } \\
\text { Plumeria alba L. } \\
\text { Thevetia peruviana (Pers.) } \\
\text { Schum. }\end{array}$} & Oleandro & 2 & 2 & 1 & 2 \\
\hline & & Jasmim-manga & 2 & 2 & 2 & - \\
\hline & & Chapéu-de-napoleão & 2 & - & 1 & - \\
\hline Araceae & $\begin{array}{l}\text { Zantedeschia aethiopica } \\
\text { (L.) Spreng }\end{array}$ & Copo-de-leite & 2 & 1 & - & \\
\hline \multirow[t]{2}{*}{ Araliaceae } & $\begin{array}{l}\text { Polyscias guilfoylei L.H. } \\
\text { Bailey. }\end{array}$ & \multirow[t]{2}{*}{ Árvore-da-felicidade } & \multirow[t]{2}{*}{2} & 1 & - & - \\
\hline & Schefflera sp. & & & - & 1 & 1 \\
\hline Arecaceae & $\begin{array}{l}\text { Roystonea oleracea (Jacq.) } \\
\text { O.F.Cook }\end{array}$ & Palmeira-real & 2 & 1 & - & 2 \\
\hline Bignoniaceae & $\begin{array}{l}\text { Jacaranda brasiliana } \\
\text { (Lam.) Pers. }\end{array}$ & $\begin{array}{l}\text { Jacarandá-boca-de- } \\
\text { sapo }\end{array}$ & 1 & - & 1 & $\begin{array}{l}1 \\
3 \\
\end{array}$ \\
\hline \multirow{5}{*}{ Bignoniaceae } & \multirow{5}{*}{$\begin{array}{l}\text { Jacaranda mimosaefolia D. } \\
\text { Don } \\
\text { Spathodea campanulata P. } \\
\text { Beauv. } \\
\text { Stenolobium stans (Juss.) } \\
\text { Seem } \\
\text { Tabebuia impetiginosa } \\
\text { (Mart. ex DC.) Standl. } \\
\text { Tabebuia roseo-alba (Ridl.) } \\
\text { Sandwith }\end{array}$} & Jacarandá-mimoso & 2 & - & - & 1 \\
\hline & & Mijo-de-macaco & 2 & - & 2 & - \\
\hline & & Ipêzinho & 2 & - & - & 7 \\
\hline & & Ipê-rosa & 1 & 89 & 7 & 7 \\
\hline & & Ipê-branco & 1 & - & 2 & 1 \\
\hline Cactaceae & Opuntia ficus-indica & Cacto & 2 & 1 & 1 & - \\
\hline Chrysobalanaceae & $\begin{array}{l}\text { Licania tomentosa (Benth.) } \\
\text { Fritsch. }\end{array}$ & Oiti & 1 & 239 & 121 & 3 \\
\hline Combretaceae & Terminalia catappa L. & Amendoeira & 2 & 52 & 8 & \\
\hline Cupressaceae & Cupressus sp. & Pinheiro & & 1 & - & - \\
\hline Euphorbiaceae & Joannesia princeps Vell. & Boleira & 1 & - & - & 6 \\
\hline \multirow{8}{*}{ Fabaceae } & Bauhinia variegata & Pata-de-vaca & 2 & 4 & 50 & $\begin{array}{l}1 \\
8\end{array}$ \\
\hline & Caesalpinia echinata Lam. & Pau-brasil & 1 & 2 & 1 & \\
\hline & Caesalpinia pluviosa DC. & Sibipiruna & 1 & 168 & 112 & $\begin{array}{l}2 \\
5\end{array}$ \\
\hline & \multirow{5}{*}{$\begin{array}{l}\text { Caesalpinia pulcherrima } \\
\text { (L.) SW. } \\
\text { Delonix regia (Bojer ex } \\
\text { Hook.) Raf. } \\
\text { Parkinsonia aculeata L. } \\
\text { Cassia ferruginea (Schrad.) } \\
\text { Schrad. ex DC. } \\
\text { Bauhinia sp. }\end{array}$} & $\begin{array}{l}\text { Flamboyant-de- } \\
\text { jardim }\end{array}$ & 2 & 2 & 2 & - \\
\hline & & \multirow[t]{2}{*}{ Flamboyant } & 2 & 3 & 7 & $\begin{array}{l}2 \\
9\end{array}$ \\
\hline & & & 2 & 1 & - & - \\
\hline & & Canafístula & 1 & - & 4 & 5 \\
\hline & & Pata-de-vaca & & - & - & 3 \\
\hline
\end{tabular}




\begin{tabular}{|c|c|c|c|c|c|c|c|}
\hline \multirow{2}{*}{ Família } & \multirow{2}{*}{ Espécie } & \multicolumn{2}{|l|}{ Nome popular } & \multicolumn{4}{|c|}{ Indiv./amb. } \\
\hline & & \multirow{2}{*}{\multicolumn{2}{|c|}{ Sena }} & & M. $\mathbf{P}$ & M. Pq & $\mathbf{P}$ \\
\hline \multirow{13}{*}{ Fabaceae } & $\begin{array}{l}\text { Senna multijuga (L.C.Rich.) } \\
\text { H.S.Irwin \& Barneby }\end{array}$ & & & 1 & - & - & 5 \\
\hline & Senna siamea (Lam.) H.S. & \multicolumn{2}{|l|}{ Cássia-do-sião } & 2 & 1 & - & - \\
\hline & Acacia mangium Willd. & \multicolumn{2}{|c|}{ Acácia-australiana } & 2 & - & 2 & 1 \\
\hline & $\begin{array}{l}\text { Albizia lebbeck (L.) } \\
\text { Bentham }\end{array}$ & \multicolumn{2}{|l|}{ Albizia } & 2 & 2 & 2 & 1 \\
\hline & $\begin{array}{l}\text { Leucaena leucocephala } \\
\text { (Lam.) de Wit. }\end{array}$ & \multirow{2}{*}{\multicolumn{2}{|c|}{ Leucena }} & 2 & - & 6 & 6 \\
\hline & Mimosa sp. & & & & 1 & - & - \\
\hline & $\begin{array}{l}\text { Pithecellobium dulce } \\
\text { (Roxb.) Benth. }\end{array}$ & \multicolumn{2}{|l|}{ Ingá-doce } & 2 & - & 1 & - \\
\hline & Prosopis juliflora (Sw.) DC. & \multirow{2}{*}{\multicolumn{2}{|c|}{ Algaroba }} & 2 & 1 & 36 & 7 \\
\hline & & & & & 1 & & $1^{-}$ \\
\hline & $\begin{array}{l}\text { Andira cujabensis Benth. } \\
\text { Erythrina sp. }\end{array}$ & Morcego & 1 & $\overline{1}$ & & $\begin{array}{l}- \\
-\end{array}$ & 1 \\
\hline & $\begin{array}{l}\text { Machaerium hirtum (Vell.) } \\
\text { Stellfeld }\end{array}$ & Pico-de-pato & 1 & - & & 1 & - \\
\hline & Machaerium opacum Vogel & Jacarandá-do- & 1 & - & & - & 2 \\
\hline & $\begin{array}{l}\text { Poecilanthe parviflora } \\
\text { Benth. }\end{array}$ & $\begin{array}{l}\text { Coração-de- } \\
\text { negro }\end{array}$ & 2 & - & & 3 & 5 \\
\hline Lamiaceae & Lavandula angustifolia Mill. & Lavanda & 2 & 1 & & - & - \\
\hline Lauraceae & Persea gratissima Gaertn. & Abacateiro & 2 & 1 & & - & - \\
\hline Lythraceae & Punica glanatum $\mathrm{L}$. & Romã & 2 & - & & 1 & - \\
\hline Malvaceae & Ceiba speciosa (A.St.-Hil.) & Paineira & 1 & 3 & & 1 & 1 \\
\hline \multirow{5}{*}{ Malvaceae } & $\begin{array}{l}\text { Dombeya wallichii (Lindl.) } \\
\text { Benth }\end{array}$ & Aurora & 2 & 1 & & - & - \\
\hline & Guazuma ulmifolia Lam. & Mutamba & 1 & - & & 1 & 2 \\
\hline & Hibiscus rosa-sinensis Linn & Hibisco & 2 & 1 & & 1 & - \\
\hline & Hibiscus sp. & Hibisco & 2 & 1 & & 1 & - \\
\hline & $\begin{array}{l}\text { Pseudobombax } \\
\text { grandiflorum (Cav.) } \\
\text { A.Robyns }\end{array}$ & Embiruçu & 1 & - & & 1 & - \\
\hline Meliaceae & Cedrela fissilis Vell. & Cedro & 1 & - & & 2 & - \\
\hline \multirow{5}{*}{ Moraceae } & Ficus benjamina L. & $\begin{array}{l}\text { Figueira- } \\
\text { benjamin }\end{array}$ & 2 & 321 & & 57 & 4 \\
\hline & $\begin{array}{l}\text { Ficus macrocarpa H. Lév. } \\
\text { \& Vaniot }\end{array}$ & Ficus & 2 & 1 & & - & - \\
\hline & Ficus microcarpa L.f. & Ficus-retusa & 2 & 2 & & 2 & - \\
\hline & Ficus sp. & & & 2 & & - & 2 \\
\hline & Morus nigra L. & Amora-preta & 2 & 1 & & - & - \\
\hline \multirow{5}{*}{ Myrtaceae } & $\begin{array}{l}\text { Callistemon viminalis G. } \\
\text { Don ex Loud. }\end{array}$ & Calistemo & 2 & 1 & & - & - \\
\hline & Eugenia dysenterica DC. & Cagaita & 1 & - & & - & 1 \\
\hline & Myrtus communis L. & Murta-comum & 2 & 2 & & - & - \\
\hline & Psidium guajava L. & Goiaba & 1 & - & & - & 1 \\
\hline & $\begin{array}{l}\text { Syzygium jambolanum } \\
\text { (Lam.) }\end{array}$ & Jamelão & 2 & 1 & & - & - \\
\hline Nyctaginaceae & $\begin{array}{l}\text { Bougainvillea glabra } \\
\text { Choisy }\end{array}$ & Buganvile & 2 & 2 & & - & 3 \\
\hline Rubiaceae & Genipa americana L. & Jenipapo & 1 & - & & - & 1 \\
\hline Rutaceae & Murraya exotica L. & Jasmin-laranja & 2 & 24 & & 31 & - \\
\hline Sapindaceae & $\begin{array}{l}\text { Dilodendron bipinnatum } \\
\text { Radlk. }\end{array}$ & Mamoninha & 1 & - & & 1 & 1 \\
\hline
\end{tabular}




\begin{tabular}{|c|c|c|c|c|c|c|}
\hline \multirow{2}{*}{ Família } & \multirow{2}{*}{ Espécie } & \multirow{2}{*}{$\begin{array}{l}\text { Nome } \\
\text { popular }\end{array}$} & \multirow{2}{*}{0} & \multicolumn{3}{|c|}{ Indiv./amb. } \\
\hline & & & & M. P & M. Pq & $\mathbf{P}$ \\
\hline Verbenaceae & Duranta repens Linn & Pingo-de-ouro & 2 & 1 & 2 & - \\
\hline \multirow{6}{*}{ Não identificadas } & sp. 1 & & & 1 & - & - \\
\hline & sp. 2 & & & 1 & - & - \\
\hline & sp. 3 & & & 1 & - & - \\
\hline & sp. 4 & & & - & - & 1 \\
\hline & sp. 5 & & & 1 & - & - \\
\hline & sp. 6 & & & 1 & - & - \\
\hline Abundância & 1605 & & & 951 & 486 & 168 \\
\hline Riqueza & 73 & & & 46 & 38 & 33 \\
\hline
\end{tabular}

Dez espécies foram comuns aos três ambientes estudados: Albizia lebbeck, Bauhinia variegata, Caesalpinia pluviosa, Ceiba speciosa, Delonix regia, Ficus benjamina, Licania tomentosa, Nerium oleander, Prosopis juliflora e Tabebuia impetigionosa. Destas espécies, cinco pertencem à família Fabaceae, que foi a mais representativa com 11 espécies e 186 indivíduos no bairro Major Prates, 11 espécies e 225 indivíduos no Morada do Parque e 13 espécies e 105 indivíduos nas praças, somando 19 espécies e 519 indivíduos (Figura 1). Este resultado mostra a mesma tendência da arborização do campus da Universidade de Brasília, em que a família Fabaceae, perfez 32\% da distribuição das famílias botânicas (KURIHARA et al., 2005).

Figura 1. Famílias botânicas mais abundantes da arborização de três ambientes na cidade de Montes Claros, MG

Figure 1. Most abundant botanic families of the urban afforestation in three areas in Montes Claros City, MG

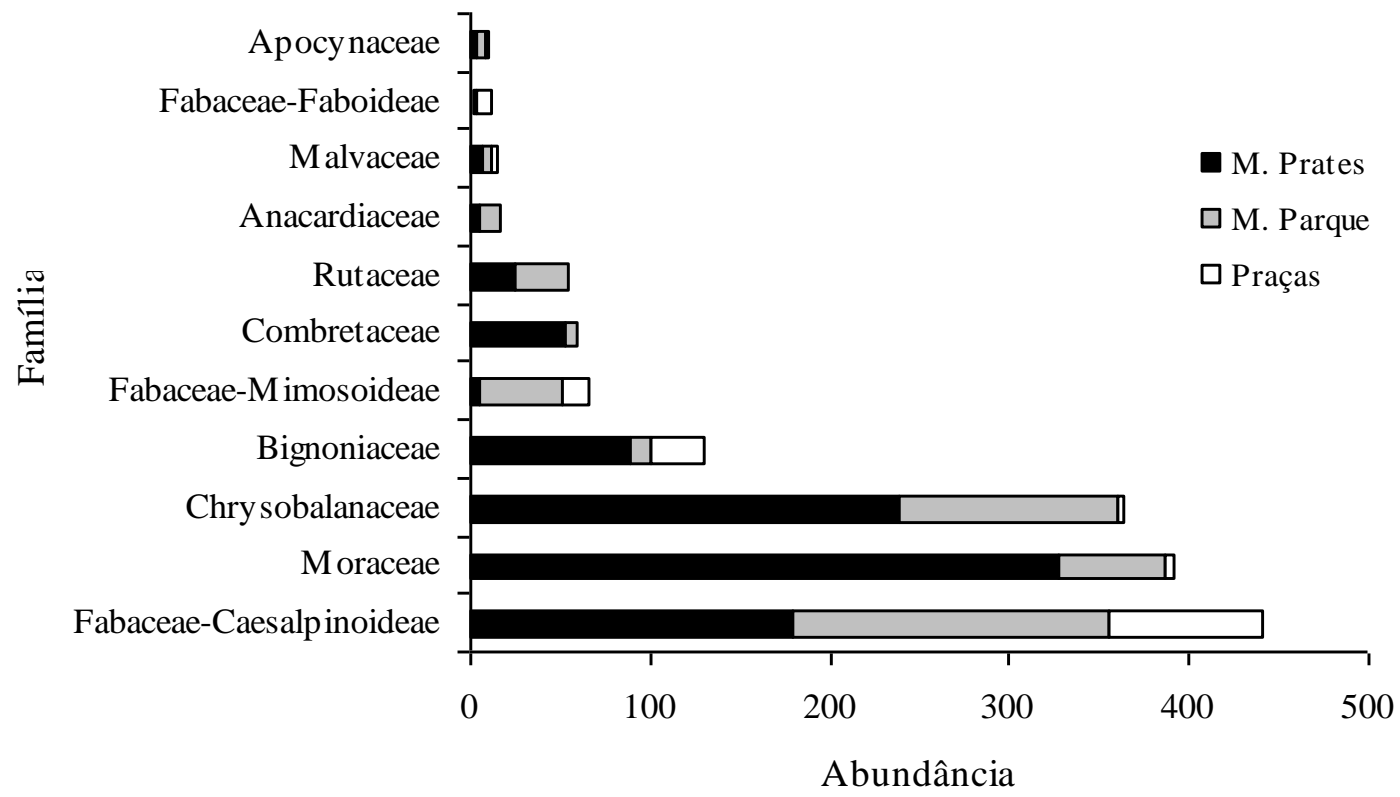


Caesalpinia pluviosa foi a espécie que mais contribuiu com a representatividade da família Fabaceae, apresentando 305 indivíduos nos três ambientes estudados (Figura 2). Silva Filho et al. (2002), trabalhando nos passeios do bairro Mansour em Uberlândia (MG), encontraram 30 espécies, sendo $L$. tomentosa e $F$. benjamina as mais abundantes, corroborando com este trabalho e indicando que estas espécies também são utilizadas na arborização em outros locais. Para Kurihara et al. (2005), C. ferrea e C. pluviosa estavam entre as 10 espécies mais frequentes encontradas na arborização do campus da Universidade de Brasília (DF).

Figura 2. Espécies arbóreas/arbustivas mais abundantes na arborização de três ambientes na cidade de Montes Claros, MG

Figure 2. Species arboreal and shrub most abundant in the urban afforestation of three environments in Montes Claros City, MG

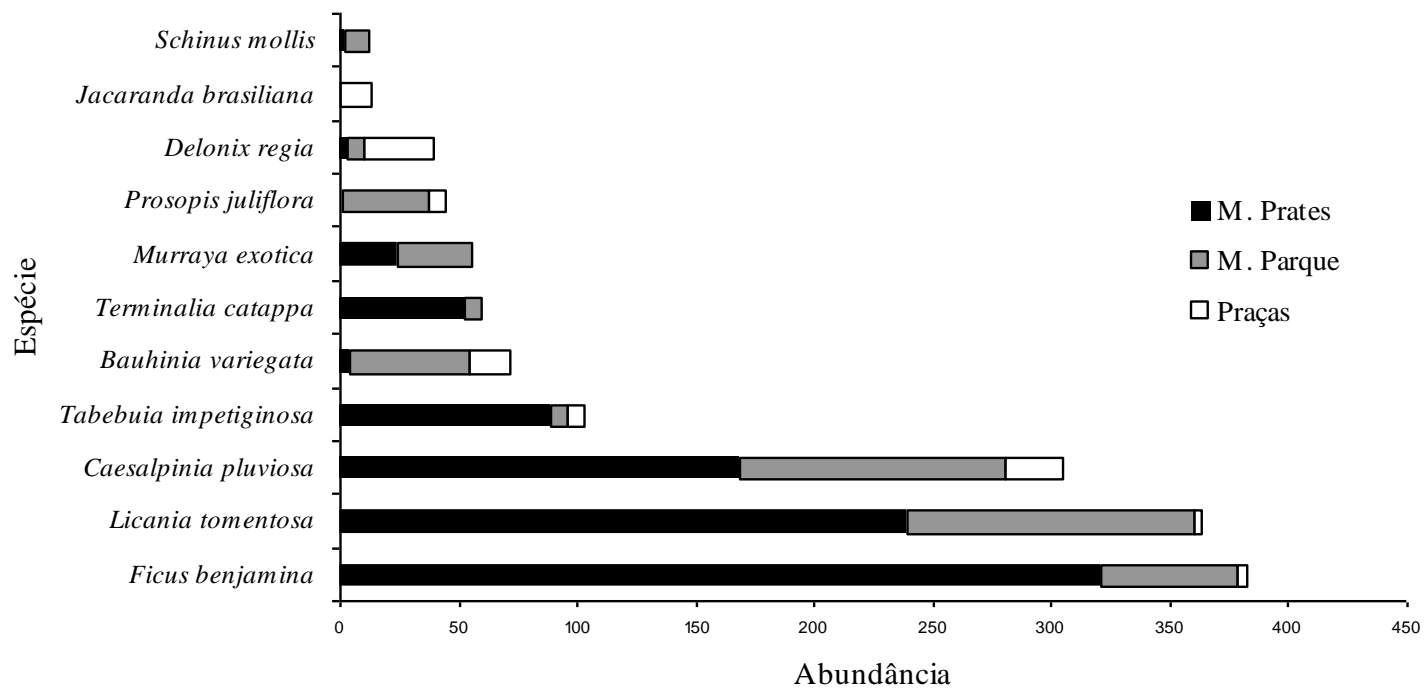

Quanto à diversidade de espécies, as praças apresentaram o maior índice $\left(H^{\prime}=\right.$ $2,91)$, seguida dos bairros Morada do Parque $\left(H^{\prime}=2,34\right)$ e Major Prates $\left(H^{\prime}=1,89\right)$. O baixo valor de diversidade encontrado no bairro Major Prates provavelmente ocorreu devido a grande dominância de algumas espécies como $F$. benjamina (321), L. tomentosa (239), $C$. pluviosa (168) e T. impetiginosa (89), que juntas perfizeram $86 \%$ dos indivíduos amostrados (Figura 2). Esses valores de diversidade foram semelhantes àqueles encontrados por Meneghetti (2003) em dois bairros da orla marítima do município de Santos (SP) $\left(H^{\prime}=2,63\right)$. Entretanto, os valores de diversidade deste estudo contrariam Milano e Dalcin (2000) que propuseram uma adequada distribuição das espécies no planejamento da arborização. De forma semelhante, Grey e Deneke (1978) recomendam uma frequência de 10 a 15\%, para cada espécie utilizada. Deste modo, a dominância discrepante de quatro espécies nestes 
três ambientes sugere que a arborização praticada no passado não seguiu os paramentos indicados por estes autores.

Quando analisada a altura das plantas, percebe-se uma diferença significativa em relação aos três ambientes $(\mathrm{gl}=2 ; \mathrm{F}=21,054 ; \mathrm{p}<0,001, \mathrm{n}=1605)$. Nas praças foram encontradas as maiores árvores, em relação àquelas dos bairros Major Prates e Morada do Parque (Tabela 2). Em relação às espécies, Jacaranda brasiliana apresentou maior porte (Tabela 3). Segundo Meneghetti (2003), a deficiência de oxigênio no solo, pode ser o fator determinante no crescimento relativamente menor das árvores em ambiente pavimentado. Provavelmente, o menor porte das árvores dos bairros em relação às praças pode ser consequência da compactação oriunda da pavimentação, o que não permite a penetração da água, inibindo o crescimento da planta, além da diminuição da área de desenvolvimento do sistema radicular. Este fato pode ser corroborado com o histórico levantado nos bairros, onde é colocado pelos moradores que a arborização aconteceu no período de criação dos mesmos, com ocorrência eventual da substituição de alguns indivíduos ao longo do tempo, no bairro Major Prates. Outro fator que pode também contribuir com estes valores é a utilização de podas nas árvores localizadas nos bairros, pois é comum a ocorrência de contenção daqueles indivíduos de maior porte, e que atingem a fiação elétrica, através de podas periódicas. Tais eventos não foram observados nas praças, que não possuem áreas pavimentadas próximas às plantas e não sofrem podas de contenção.

Tabela 2. Valores médios ( \pm desvio padrão) dos parâmetros estruturais dos indivíduos amostrados na arborização de três ambientes em Montes Claros, MG. $\mathrm{n}=$ amostra

Table 2. Average values ( \pm standard deviation) of the urban afforestation parameters obtained of the individuals sampled in three environments in Montes Claros, MG. $\mathrm{n}=$ sample

\begin{tabular}{|c|c|c|c|}
\hline \multirow{2}{*}{ Parâmetros } & \multicolumn{3}{|c|}{ Ambientes } \\
\hline & Major Prates & Morada do Parque & Praças \\
\hline Altura (m) & $\begin{array}{l}\bar{X}=5,82 \pm 4,31 \\
n=951\end{array}$ & $\begin{array}{l}\bar{X}=6,01 \pm 2,40 \\
n=486\end{array}$ & $\begin{array}{l}\bar{X}=7,81 \pm 2,67 \\
\mathrm{n}=168\end{array}$ \\
\hline Tamanho de copa $(\mathrm{cm})$ & $\begin{array}{l}\bar{X}=106,43 \pm 87,53 \\
n=951\end{array}$ & $\begin{array}{l}\bar{X}=123,37 \pm 99,85 \\
n=486\end{array}$ & $\begin{array}{l}\bar{X}=199,16 \pm 183,71 \\
n=168\end{array}$ \\
\hline $\begin{array}{l}\text { Tamanho da rachadura } \\
\text { no passeio }\end{array}$ & $\begin{array}{l}\bar{X}=5,10 \pm 5,63 \\
n=377\end{array}$ & $\begin{array}{l}\bar{X}=1,22 \pm 0,90 \\
\mathrm{n}=222\end{array}$ & - \\
\hline Distância da fiação & $\begin{array}{l}\bar{X}=0,55 \pm 2,07 \\
n=452\end{array}$ & $\begin{array}{l}\bar{X}=0,87 \pm 1,83 \\
n=245\end{array}$ & - \\
\hline $\begin{array}{l}\text { Espaçamento } \\
\text { indivíduos }\end{array}$ & $\begin{array}{l}\bar{X}=29,28 \pm 43,70 \\
n=951\end{array}$ & $\begin{array}{l}\bar{X}=19,93 \pm 24,49 \\
n=486\end{array}$ & - \\
\hline Área livre do passeio & $\begin{array}{l}\bar{X}=12,60 \pm 20,06 \\
n=950\end{array}$ & $\begin{array}{l}\bar{X}=15,28 \pm 32,33 \\
n=486\end{array}$ & - \\
\hline
\end{tabular}


Tabela 3. Valores médios ( \pm desvio padrão) dos parâmetros obtidos das espécies mais abundantes amostradas na arborização de três ambientes na cidade de Montes Claros, MG

Table 3. Average values ( \pm standard deviation) of the urban afforestation parameters obtained for the most abundant species sampled in three environments in Montes Claros City, MG

\begin{tabular}{llllllc}
\hline \multirow{2}{*}{ Espécies } & \multicolumn{7}{c}{ Parâmetros } \\
\cline { 2 - 7 } & Altura (m) & DF $(\mathbf{m})$ & ALP (cm) & TC (cm) & TRP (m) & El (m) \\
\hline Ficus benjamina & $4,7 \pm 1,9$ & $0,1 \pm 1,8$ & $11,5 \pm 17,1$ & $72,8 \pm 71,7$ & $4,5 \pm 5,3$ & $30,8 \pm 40,4$ \\
Licania tomentosa & $6,3 \pm 2,2$ & $1,1 \pm 2,0$ & $11,5 \pm 29,1$ & $96,2 \pm 58,4$ & $3,1 \pm 3,4$ & $27,1 \pm 41,2$ \\
Caesalpinia pluviosa & $6,8 \pm 1,9$ & $1,3 \pm 1,5$ & $12,0 \pm 23,3$ & $166,0 \pm 85,5$ & $4,2 \pm 5,8$ & $25,9 \pm 35,1$ \\
Tabebuia impetiginosa & $6,8 \pm 1,8$ & $2,1 \pm 0,8$ & $28,8 \pm 32,7$ & $170,6 \pm 109,6$ & $0,9 \pm 0,2$ & $11,6 \pm 17,2$ \\
Bauhinia variegata & $6,4 \pm 1,5$ & $1,6 \pm 1,3$ & $6,3 \pm 11,8$ & $123,0 \pm 53,2$ & $1,2 \pm 0,9$ & $24,9 \pm 21,3$ \\
Terminalia catappa & $8,7 \pm 8,3$ & $1,5 \pm 1,6$ & $12,5 \pm 17,3$ & $209,3 \pm 127,1$ & $4,0 \pm 5,8$ & $28,3 \pm 29,8$ \\
Murraya exotica & $4,0 \pm 13,2$ & $1,6 \pm 2,5$ & $14,8 \pm 12,8$ & $11,7 \pm 12,1$ & $1,8 \pm 0$ & $24,4 \pm 30,1$ \\
Prosopis fuliflora & $7,4 \pm 2,0$ & $0,8 \pm 2,4$ & $0,6 \pm 1,4$ & $218,5 \pm 123,8$ & $1,1 \pm 0,5$ & $19,7 \pm 20,4$ \\
Delonix regia & $7,0 \pm 1,4$ & $0,4 \pm 1,4$ & $7,3 \pm 29,1$ & $218,5 \pm 149,2$ & $3,9 \pm 6,0$ & $43,4 \pm 35,5$ \\
Jacaranda brasiliana & $10,4 \pm 2,5$ & $1,5 \pm 0$ & $5,4 \pm 6,3$ & $347,4 \pm 277,9$ & $4,3 \pm 4,1$ & $56,4 \pm 31,9$ \\
Leucaena leucocephala & $10,2 \pm 4,3$ & $2,8 \pm 3,1$ & $3,5 \pm 7,7$ & $304,6 \pm 315,4$ & $1,1 \pm 0,2$ & $37,8 \pm 30,2$ \\
Schinus mollis & $3,0 \pm 1,5$ & $0,4 \pm 1,1$ & $23,8 \pm 14,1$ & $63,7 \pm 115,7$ & $1,1 \pm 0,2$ & $36,1 \pm 29,3$ \\
\hline
\end{tabular}

Foram observadas diferenças em relação às rachaduras nos passeios provenientes das raízes das plantas entre os bairros ( $\mathrm{gl}=597 ; t=10,19 ; \mathrm{p}<0,001 ; \mathrm{n}=599$ ). $\mathrm{O}$ bairro Major Prates apresentou uma média de raiz exposta, ou de rachadura no passeio, maior do que aquela apresentada no bairro Morada do Parque (Tabela 2). Provavelmente, as rachaduras maiores nos passeios do bairro Major Prates possam ser explicadas devido o tempo de exposição, ao desenvolvimento das árvores, que a pavimentação do passeio sofreu, uma vez que, o processo de urbanização desse bairro é mais antigo que o daquele feito no bairro Morada do Parque. Além disso, a maior abundância de F. benjamina (321) e Terminalia catappa (52), no bairro Major Prates em relação ao Morada do Parque (57 e 8, respectivamente), também pode ter contribuído para a discrepância na média da rachadura, visto que Meneghetti (2003) ressaltou que essas duas espécies são impróprias para o plantio, devido à exposição de suas raízes e consequente dano aos passeios. Apesar de não ter sido observada diferença significativa na área livre dos passeios entre os bairros $(\mathrm{gl}$ $=1434 ; t=-1,93 ; p>0,05 ; n=1436$ ), a ocorrência de rachaduras nos passeios podem sugerir que essa área destinada ao desenvolvimento das árvores é insuficiente, além da própria improbidade das espécies utilizadas (grande porte). 
Com relação à área de copa das árvores ( $\mathrm{gl}=2 ; \mathrm{F}=55,55 ; \mathrm{p}<0,01 ; \mathrm{n}=1605$ ), pode-se constatar maiores médias para as praças, seguidos dos bairros Major Prates e Morada do Parque (Tabela 2). As espécies que apresentaram maiores médias para a área de copa foram J. brasiliana, L. leucocephala e T. catappa (Tabela 3), o que indica que estas espécies necessitam de maior espaço para seu crescimento e, consequentemente maior espaçamento entre indivíduos (TONINI; ARCO VERDE, 2005). Neste sentido, estas espécies podem ser consideradas impróprias para plantio próximo a edificações e recomendadas em áreas abertas como praças, onde podem proporcionar sombreamento de qualidade para os seus frequentadores.

Foi evidenciada diferença entre a distância da fiação para os dois bairros estudados ( $\mathrm{gl}=1 ; t=-2,04 ; \mathrm{p}<0,05 ; \mathrm{n}=697$ ). O bairro Major Prates apresentou uma média de distância que ultrapassa a fiação menor do que aquela encontrada no Morada do Parque (Tabela 2). Estes resultados indicam que as árvores localizadas no bairro Morada do Parque possuem maior porte. O plantio de árvores de grande porte debaixo de rede elétrica provoca normalmente, conflitos das árvores com esta rede (SILVA et al., 2002), implicando em prejuízos para a população local, através da redução de áreas sombreadas e também para o município, com serviços de manutenção das redes elétricas e com a mutilação de alguns indivíduos, através de podas de contenção. Assim, a espécie Ficus benjamina, apesar de ter apresentado uma altura média inferior a cinco metros, é uma espécie de grande porte e foi observado que as copas dos indivíduos desta espécie, encontradas nas ruas, eram conduzidas por podas ou ainda eram indivíduos jovens. Portanto, considerando esta característica peculiar, F. Benjamina, não é uma espécie indicada para a arborização de ruas.

Não foi observado diferença no espaçamento entre os indivíduos nos bairros $(\mathrm{gl}=$ 1435; $t=4,38 ; \mathrm{p}>0,05)$. O bairro Major Prates obteve média de 29,27 $\mathrm{m}$ de distância entre os indivíduos e o Morada do Parque de 19,93 m (Tabela 2). De acordo com estes resultados, o bairro Major Prates, que estruturalmente apresenta largura de ruas e passeios similares ao bairro Morada do Parque apresenta um sombreamento inferior. Desta forma, apesar da maior quantidade de indivíduos amostrados no bairro Major Prates, o bairro Morada do Parque possui uma maior densidade, em virtude da menor área do bairro, menor distância entre os indivíduos e consequentemente, maior área sombreada e microclima mais ameno, em função do adensamento. 


\section{CONCLUSÕES}

A arborização nos três ambientes no município de Montes Claros/MG apresentou 1605 indivíduos arbóreos distribuídos em 73 espécies. Foram observadas diferenças para todos os parâmetros analisados, exceto para a área livre do passeio e o espaçamento entre os indivíduos. As praças apresentaram maior diversidade florística, altura e tamanho de copa dos indivíduos. Por outro lado, o bairro Major Prates apresentou maiores rachaduras nos passeios e o bairro Morada do Parque, maiores distâncias da fiação em relação às árvores plantadas. Além disto, estes resultados demonstram ainda diferenças marcantes nos padrões de arborização dos três ambientes estudados. Além da baixa ocorrência de espécies nativas da região e ausência de um prévio planejamento urbano, em relação à arborização, o que é essencial para evitar prejuízos aos serviços ambientais e ao bem estar da população.

\section{AGRADECIMENTOS}

À FAPEMIG pelas bolsas de BIPDT de M. D. M. Veloso e Y. R. F. Nunes e de BIC de L. L. Braga e D. O. Brandão; ao CNPq pela bolsa PIBIC de P. M. S. Rodrigues e Bolsa de Pesquisa de Y. R. F. Nunes; à UNIMONTES pelo apoio logístico; aos colegas Carlos Henrique P. Silva, Gláucia S. Tolentino, Anne Mariele Matos, Gladson R. A. Borges e Tiago Amorim pelo auxílio nos trabalhos de campo e laboratório; ao Prof. Santos D’Ângelo Neto pela identificação das espécies; e aos revisores anônimos pelas prestimosas sugestões e contribuições no manuscrito.

\section{REFERÊNCIAS}

AN UPDATE OF THE ANGIOSPERM PHYLOGENY GROUP CLASSIFICATION FOR ORDERS AND FAMILIES OF FLOWERING PLANTS: APG II. Botanical Journal of the Linnean, London, v. 141, n. 4, p. 399-436, 2003.

DANTAS, I. C.; SOUZA, C. M. C. Arborização urbana na cidade de Campina Grande - PB: Inventário e suas espécies. Revista Biologia e Ciências da Terra, Paraíba, v. 4, n. 2, p. 1 18, 2004. 
GONÇALVES, E. O.; PAIVA, H.N; GONÇALVES, W.; JACOVINE, L.A. G. Avaliação qualitativa de mudas destinadas à arborização urbana no Estado de Minas Gerais. Revista Arvore, Viçosa, v. 28, n. 4. p. 479-486, 2004.

GREY, G. W.; DENEKE, F. J. Urban forestry. New York: John Wiley, 1978. 279p.

IBGE - Instituto Brasileiro de Geografia e Estatística. Contagem da população 2007. Rio de Janeiro, 2007.

IGAM. Disponível em: < http://www.igam.mg.gov.br>. Acesso em: 08 Jul. 2007.

KREBS, C. J. Ecological methodology. New York: Harper \& Row, 1989. 654p.

KURIHARA, D. L.; IMAÑA-ENCINAS, J.; ELIAS DE PAULA, J. Levantamento da Arborização do Campus da Universidade de Brasília. Cerne, Lavras, v. 11, n. 2. p. 127-136, 2005.

MENEGUETTI, G. I. P. Estudo de dois métodos de amostragem para inventário da arborização de ruas dos bairros da orla marítima do município de Santos-SP. 2003. 100 p. Dissertação (Mestrado em Recursos Florestais) - Escola Superior de Agricultura "Luiz de Queiroz", Piracicaba.

MILANO, M.; DALCIN, E. Arborização de vias públicas. Rio de Janeiro: Light, 2000. 226p.

OLIVEIRA, R. M. F. Relatório de caracterização da região administrativa do bairro Major Prates. Secretaria Municipal de Planejamento e Coordenação, Gerência de Planejamento e Coordenação, Divisão de Coordenação, Prefeitura Municipal de Montes Claros, Montes Claros, MG, 2003, 16p.

ROCHA, R. T.; LELES, P. S. S.; OLIVEIRA NETO, S. N. Arborização em vias públicas em Nova Iguaçu, RJ: o caso dos bairros Rancho Novo e Centro. Revista Árvore, Viçosa, v. 28, n. 4, p. 599- 607, 2004.

RODRIGUES, C. A. G.; BEZERRA, B. C.; ISHII, I. H.; CARDOSO, E. L.; SORIANO, B. M. A.; OLIVEIRA, $H$. Arborização urbana e produção de mudas de essências florestais nativas em Corumbá, MS. Corumbá: Embrapa Pantanal, 2002. 26p.

SILVA, E. M.; SILVA, A. M.; MELO, P. H.; BORGES, S. S.; LIMA, S. C. Estudo da arborização urbana do bairro Mansour, na cidade de Uberlândia-MG. Caminhos de Geografia, Uberlândia, v. 3, n. 5, p. 73-83, 2002. 
SILVAFILHO, D. F.; PIVETTA, K. F. L.; COUTO, H. T. Z. \& POLIZEL, J. L. Indicadores de floresta urbana a partir de imagens aéreas multiespectrais de alta resolução. Scientia Forestalis, São Paulo, n. 67, p. 88-100, 2005.

SILVA FILHO, D. F.; PIZETTA, P. U. C.; ALMEIDA, J. B. S. A.; PIVETTA, K. F. L.; FERRAUDO, A. S. Banco de dados relacional para cadastro, avaliação e manejo da arborização em vias públicas. Revista Árvore, Viçosa, v. 26, n. 5, p. 629-642, 2002.

SOLERA, M.; MEIRERZ, F. R.; CARDOSO, J. D.; DONASSOLO, V. C.; OLIVEIRA, P. J. S.; HEFLER, S. M. Diagnóstico da Arborização Urbana no município de Toledo, Paraná, Brasil. In: 56 CONGRESSO NACIONAL DE BOTÂNICA, 2005. Anais Parte I. Anais do $56^{\circ}$ Congresso Nacional de Botânica. Curitiba.

TONINI, H.; ARCO VERDE, M. F. Morfologia da copa para avaliar o espaço vital de quatro espécies nativas da Amazônia. Pesquisa Agropecuária Brasileira, Brasília, v. 40, n. 7, p. 633-638, 2005.

ZANIN, E. M.; SANTOS, J. E.; HENKE-OLIVEIRA, C. Environmental analysis and zoning for an urban park management purpose. Brazilian Archives of Biology and Technology, Curitiba, v. 48, n. 4, p. 647-655, 2005.

ZAR, J. H. Biostatistical analysis. New Jersey: Prentice-Hall, 1996. 662p. 